\title{
Role of Perioperative Echocardiography Leadership in a Tertiary University Hospital
}

\author{
${ }^{1}$ Kathirvel Subramaniam, ${ }^{2}$ Balachundhur Subramaniam
}

\begin{abstract}
Echocardiography has been shown to improve the perioperative outcomes of surgical and critically ill patients. Several modalities of echocardiography (transthoracic, transesophageal, epicardial and epiaortic) are being utilized clinically for the hemodynamic management of surgical patients. They can be collectively described as perioperative echocardiography (PEC). Because of such a wider scope of practice in perioperative echocardiography, there is a need for leadership to maintain equipment, good clinical practice, education, research, quality, documentation, billing and reimbursement. American Society of Echocardiography (ASE) and Society of Cardiovascular Anesthesiologists (SCA) published guidelines for performance, reporting, education and quality improvement in PEC. The major role of echocardiography leadership is to ensure PEC team follows the guidelines published by ASE/SCA in their practice and utilize the potential of the various modalities to the benefit and safety of their patients. This article explores the key roles of the director for perioperative echocardiography service at a tertiary university hospital.
\end{abstract}

Keywords: Perioperative echocardiography, Leadership role, University hospital.

How to cite this article: Subramaniam K, Subramaniam B. Role of Perioperative Echocardiography Leadership in a Tertiary University Hospital. J Perioper Echocardiogr 2015;3(1):4-16.

\section{Source of support: Nil}

Conflict of interest: None declared

\section{INTRODUCTION}

Intraoperative transesophageal echocardiography (TEE) has become an integral component of cardiac surgery. Improved outcomes have been reported with the use of TEE by anesthesiologists for diagnostic purposes

${ }^{1}$ Visiting Associate Professor, ${ }^{2}$ Associate Professor and Director

${ }^{1}$ Department of Anesthesiology, University of Pittsburgh Pittsburgh, Pennsylvania, USA

${ }^{2}$ Harvard Medical School; Center for Anesthesia Research Excellence, Beth Israel Deaconess Medical Center, Boston Massachusetts, USA

Corresponding Author: Kathirvel Subramaniam, Visiting Associate Professor, Department of Anesthesiology, University of Pittsburgh, Pittsburgh, Pennsylvania 15213, USA, Phone: 4126475635, e-mail: subramaniamk@upmc.edu during cardiac surgery. ${ }^{1,2}$ Intraoperative use of 'rescue' TEE has also been shown to be useful in the diagnosis of hemodynamic instability during non-cardiac surgery. ${ }^{3,4}$ Transesophageal echocardiography is also used for intraoperative hemodynamic monitoring of patients with cardiac disease undergoing high-risk non-cardiac surgical procedures (e.g. abdominal and descending aortic aneurysm surgery). ${ }^{5,6}$ Transesophageal echocardiography has also proven its usefulness in the care of critically ill patients in the postoperative period. ${ }^{7,8}$ Epicardial and epiaortic echocardiography are other modalities of intraoperative echocardiography, which can be used to guide the cardiac surgical procedures..$^{9,10}$ Transthoracic echocardiography (TTE) is used by critical care specialists in intensive care unit (ICU) for the diagnosis of hypoxemia and hemodynamic instability and also to guide interventional procedures. ${ }^{11,12}$ The role of PEC has grown up extensively in the recent years and there is clear need for effective leadership to maintain standards in echocardiography practice. This article explores the key roles of the director for perioperative echocardiography service at a tertiary university hospital.

\section{CLINICAL PRACTICE GUIDELINES}

American Society of Echocardiography and SCA published practice guidelines for intraoperative TEE, epiaortic and epicardial echocardiography..$^{13}$ Any cardiac surgery or thoracic aortic surgery is automatically an indication for intraoperative TEE. The recommendation is based on the evidence that TEE can confirm and refine the preoperative echocardiographic findings, identify new pathological process in the heart and assess the results of surgical interventions. Intraoperative TEE is also indicated in patients undergoing catheter based intracardiac interventions, such as atrial septal device (ASD) closure, transcatherter aortic valve (AV) replacement, mitraclip application and atrial appendage closure. During noncardiac surgery, TEE is indicated to establish the definite diagnosis for hemodynamic instability (e.g. pulmonary thromboembolism during major orthopedic surgery). Transesophageal echocardiography is also indicated when nature of surgery or patient's medical condition is anticipated to result 
in significant hemodynamic, respiratory or neurologic compromise. Sitting craniotomy in a patient with history of patent foramen ovale (PFO) is an example, where TEE monitoring may be indicated to prevent neurologic dysfunction. Elderly patient with severe aortic stenosis (AS) undergoing hip replacement surgery is an example of a patient at risk for perioperative hemodynamic compromise. When it comes to postoperative care and critical care units, TTE is indicated initially to identify the etiology for hemodynamic compromise. Transesophageal echocardiography may be indicated if other modalities are not able to provide the definite diagnosis.

Standard 20 TEE views have been replaced by 28 views based on expanded indications and technology. ${ }^{14}$ Threedimensional echocardiographic examination (3DTEE) is added to provide guidance on the use of this technology for cardiac surgical patients. ${ }^{15}$ In addition, several guidelines have been published to help the perioperative echocardiographer with chamber quantification, native and prosthetic valve examination and hemodynamic calculations (Table 1). ${ }^{14,16,17}$
Transesophageal echocardiography is safe and complications are rare but can have serious consequences. ${ }^{18}$ Each department should have a written policy on safety guidelines for the use of TEE probe. The policy adopted by the Department of Anesthesiology, University of Pittsburgh Medical Center (UPMC) is described in Table 2. In patients with esophageal or gastric disease, benefits of doing TEE should outweigh risks (Table 3). Risk-benefit ratio should be discussed with the surgical team and the patient in detail and documented in the anesthesia record before the procedure. Root cause analysis should be performed by thorough investigation of all TEE related complications with the aim of implementing changes in practice to avoid such events and bad outcomes in future. Perioperative echocardiography director should make his team aware of the practice guidelines, update the changes in guidelines to keep his team up to date and facilitate teaching the guidelines to the trainees so that patient safety and quality are optimized by evidence based medical practice.

Table 1: Published guidelines for the use of perioperative echocardiography

- Guidelines for a comprehensive TEE examination (SCA/ASE, updated September 2013)

- Basic TEE examination-A consensus statement (SCA/ASE)

- Recommendations for evaluation of native and prosthetic valves with echocardiography and doppler ultrasound

- Recommendations for Chamber Quantification: A Report from the ASE's guidelines and standards committee and the chamber Quantification Writing Group, developed in conjunction with the European association of echocardiography (EAE), a branch of the European society of cardiology (ESC)

- EAE/ASE recommendations for image acquisition and display using three-dimensional echocardiography

- Guidelines for ultrasound guided central venous cannulation (ASE/SCA)

- Guidelines for performance of a comprehensive intraoperative epiaortic echocardiographic examination

- Guidelines for performance of a comprehensive epicardial echocardiographic examination

- Practice guidelines for perioperative TEE (ASA/SCA Task force)

- Task force guidelines for training in perioperative TEE (ASE/SCA)

- Recommendations for a standardized report for adult perioperative echocardiography (ASE/SCA)

- Recommendations and guidelines for continuous quality improvement in perioperative echocardiography (SCA/ASE)

Table 2: University of Pittsburgh Medical Center safety policy for TEE probe management in perioperative TEE

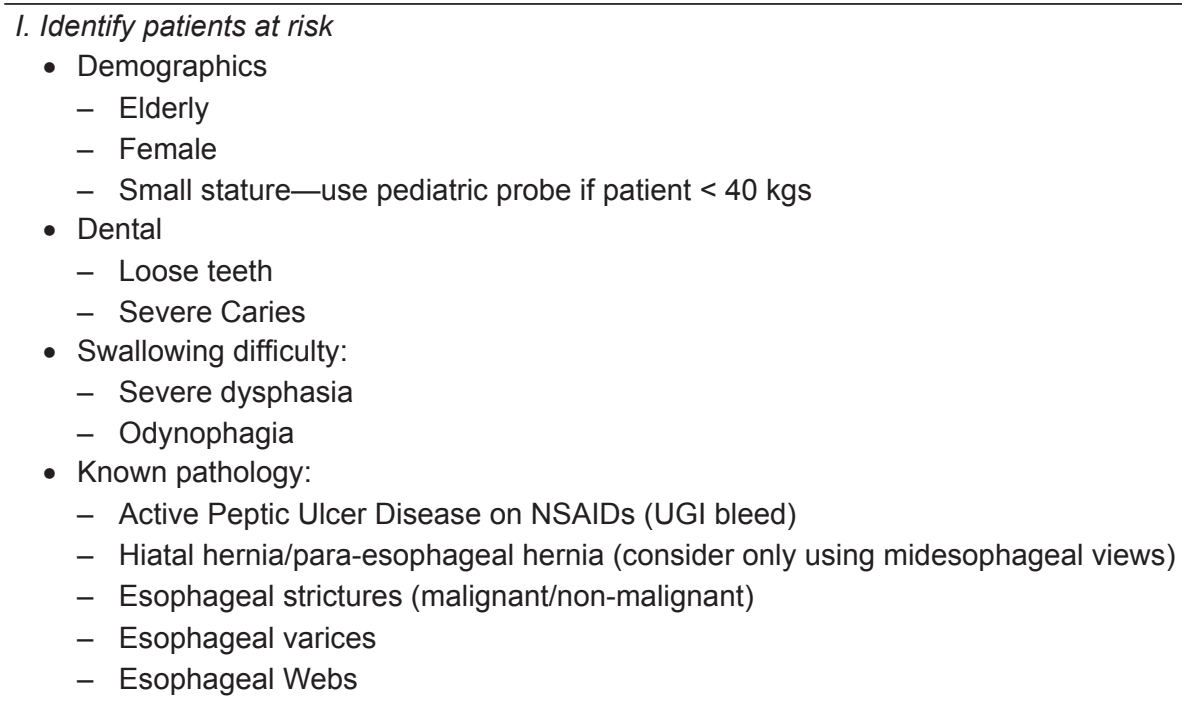

Contd... 
Contd...

- Vascular rings impinging on the esophagus

- Zenker's diverticulum

- Previous gastric/esophageal surgery:

- Esophagectomy

- Nissen fundoplication

- Bariatric surgery

- Partial Gastrectomy, Billroth, etc. a. Consider ME views only

- Prior esophageal instrumentation:

- TEE in days preceding OR

- Difficulty inserting probe by cardiology

- If risk factors for injury high, then consider consulting Thoracic surgery/GI service for EGD. Insertion could be achieved with the assistance of an overtube. Might consider aborting an elective case.

- Known esophageal Injury: Consider not doing the case until esophageal issue resolved. Consider doing case without TEE.

II. Guidelines to Minimize Esophageal Injury

A. TEE use:

- TEE examinations are limited to physicians with privileges to perform these exams and physicians in the process of pursuing this certification

- Pre-insertion:

- Surgical service to screen patient prior to surgery; if found to be at significant risk patient should have thoracic surgery/GI consult to assess, investigate, and treat

- Screen for patients at risk in anesthesiology $\mathrm{H}$ and $\mathrm{P}$

- Check TEE probe tip for imperfections

- Make sure that probe controls are not locked

- Insertion:

- Attending supervised/performed

- Pay close attention to resistance to passage

- Use bite block to protect patient's teeth and equipment

- Suggested technique:

- Head in neutral position

- Slightly flexed neck

- Ensure probe is inserted in the midline

- Slow and steady pressure to advance TEE probe

- Protocol for difficult insertion:

- Should be activated if any difficulty placing TEE

- Use laryngoscope to ensure esophagus is being engaged

- If continued resistance experienced:

i. Consult thoracic surgery/GI for EGD and possible overtube placement

ii. Consider not using TEE for the case unless there is a class I indication

iii. Consider using pediatric TEE probe

iv. Consider using epicardial scanning

- Manipulating probe during exam:

- Do not leave angulation controls locked

- Always check control locks before scanning

- Probe manipulation should be limited to what is necessary to perform a diagnostic examination and/or perform intraoperative monitoring of the patient's status as clinically indicated during the procedure

- Minimize the number of times the esophagus is traversed during the case (esp. GE junction)

- Limit study to attending only -if the patient is considered high-risk for iatrogenic injury (See Section I)

- Removal of the probe:

- Ensure wheels are unlocked before removal

- Physician to remove probes

- Check probe and mouth for presence of blood

- Insert OGT to monitor for bleeding unless injury suspected

- Suspected injury or difficulty passage:

- Surgeon and CCM are to be notified

- Consider getting early GI consult to do Barium swallow/EGD

- Documentation requirements:

i. History and physical findings that confirm presence/absence of risk of injury

ii. Describe the nature of TEE probe passage

iii. Document whether a bite-block was use

iv. Document any blood found on the probe or in oropharynx 
Role of Perioperative Echocardiography Leadership in a Tertiary University Hospital

\begin{tabular}{|c|c|}
\hline Class I indications & Class II indications \\
\hline Valve repair & Valve replacement \\
\hline Congenital surgery w/ CPB & Tumors \\
\hline Endocarditis & $\begin{array}{l}\text { Increased risk of intraoperative } \\
\text { ischemia }\end{array}$ \\
\hline Ascending aortic dissection & Thrombectomy/embolectomy \\
\hline $\begin{array}{l}\text { Unstable pt. with unknown } \\
\text { cardiac function }\end{array}$ & Cardiac trauma \\
\hline Pericardial window & Cardiac aneurysm \\
\hline HOCM & $\begin{array}{l}\text { Aortic aneurysm without aortic valve } \\
\text { Thoracic transplant } \\
\text { Monitoring placement of ventricular } \\
\text { assist devices }\end{array}$ \\
\hline
\end{tabular}

\section{ECHOCARDIOGRAPHY EDUCATION}

Education in perioperative echocardiography is an essential component of any university program. Perioperative echocardiography director is fully responsible for execution of the education program. Apart from anesthesia residents and cardiothoracic (CT) anesthesia fellows, several other departments (cardiology, cardiac surgery, critical care and emergency medicine) have shown interest in learning perioperative transesophageal echocardiography (PTE) in the operating room. In addition, anesthesiologists in practice from inside and outside the institution also want to learn and obtain National Board of Echocardiography (NBE) basic PTE certification to improve their practice. Although we should promote PTE education for all, education of anesthesiology trainees is our priority. Anesthesiology residents in their third year of training (CA-3) are offered basic PTE education and CT Anesthesiology fellows are offered advanced PTE education. The two major determinants of quality PTE education are volume and variety of cardiac surgical procedures. Both of these factors clearly correlate with the exposure and training in PTE. Education in cardiology echocardiography can supplement echocardiography education but cannot reproduce acutely changing environment of operating rooms. National Board of Echocardiography has set the clinical training requirements for basic and advanced PTE certification (www.echoboards. org) (Table 4).

First, we describe the basic PTE clinical training program at our institution developed by the director of PTE education.

\section{Basic PTE Education at University of Pittsburgh Medical Center (UPMC)}

\section{Scope of Training}

According to NBE, the application of a basic PTE is limited to nondiagnostic monitoring within the customary
Table 4: National Board of Echocardiography requirements for clinical training in PTE

\begin{tabular}{llll}
\hline & $\begin{array}{l}\text { Basic } \\
\text { PTE }\end{array}$ & $\begin{array}{l}\text { Advanced } \\
\text { PTE }\end{array}$ & $\begin{array}{l}\text { PTE } \\
\text { director }\end{array}$ \\
\hline $\begin{array}{l}\text { TEE personally performed, } \\
\text { interpreted and reported under }\end{array}$ & 50 & 150 & 150 \\
$\begin{array}{l}\text { appropriate supervision } \\
\begin{array}{l}\text { TEE interpreted and reported } \\
\text { under appropriate supervision }\end{array}\end{array}$ & 150 & 300 & 450 \\
\hline
\end{tabular}

practice of anesthesiology. Because the goal of and training in basic PTE is focused on intraoperative monitoring rather than specific diagnosis, except in emergent situations, diagnoses requiring intraoperative cardiac surgical intervention or postoperative medical/surgical management must be confirmed by an individual with advanced skills in PTE or by an independent diagnostic technique. ${ }^{19}$

\section{Rotation Goals}

By the conclusion of the TEE rotation during residency, the resident will be able to achieve the goals described in Table 5 under direct supervision of the cardiac anesthesiology faculty.

\section{Basic TEE Rotation Structure}

Two months of perioperative TEE training is provided for interested residents in their CA-3 year of training. The aim is to make them eligible for basic TEE examination and certification.

\section{Preparation for the Rotation}

Residents will be provided with clear instructions, reading materials and basic lectures (available through intranet anesthesia education website) before starting this rotation. The residents are asked to go through the online simulation website http://pie.med.utoronto.ca/ TEE/TEE_content/TEE_standardViews_intro.html to get an overview and spatial orientation to standard twodimensional TEE views. On the first day of rotation, the residents spend a day at the simulation center and learn to do basic TEE views on Echocardiography simulator. Echocardiography simulation has been shown to be useful in teaching image acquisition skills for echo-naïve residents and physicians. ${ }^{20,21}$ The aim is to make the residents comfortable in doing the ASE/SCA standard basic TEE views before manipulation of the probe on patients. They are given basic lectures on the following at the simulation center as a crash course

1. Review of requirements for basic certification

2. TEE safety precautions (See Table 2)

3. Knobology and basic principles of ultrasound

Residents are given three dedicated TEE days in a week, without any direct hands on patient care 
Table 5: Basic perioperative transesophageal echocardiography education goals

\section{Patient care:}

- Ability to operate the TEE machine including the primary controls affecting the quality of the displayed data (Knobology)

- Ability to insert a TEE probe safely in the anesthetized, tracheally intubated patient

- Ability to perform a comprehensive two-dimensional TEE examination and differentiate normal from markedly abnormal cardiac structures and function

- Ability to recognize marked changes in segmental ventricular contraction indicative of myocardial ischemia or infarction (Myocardial pump function)

- Ability to recognize marked changes in global ventricular filling and ejection (volume status)

- Ability to recognize gross valvular lesions and dysfunction

- Ability to detect large pericardial effusions, pleural and mediastinal collections

- Ability to recognize air embolism and pulmonary embolism

- Ability to recognize large intracardiac masses and thrombi

- Ability to recognize the lesions in great vessels (example; traumatic rupture of aorta)

- Ability to recognize common echocardiographic artifacts

- Ability to recognize complications of perioperative echocardiograph Medical knowledge:

- Knowledge of the physical principles of echocardiographic image formation (Basic Physics)

- Knowledge of the operation of TEE machine including all controls that affect the quality of data displayed (Knobology)

- Knowledge of the equipment handling, infection control, and electrical safety associated with the techniques of perioperative echocardiography

- Knowledge of the indications, contraindications, and potential complications for perioperative echocardiography

- Knowledge of the normal tomographic anatomy as revealed by perioperative echocardiographic techniques (Spatial orientation).

- Knowledge of different echocardiographic modalities (Two-dimensional echocardiography, Color flow Doppler and Spectral Doppler echocardiography and Three dimensional echocardiography).

- Detailed knowledge of the echocardiographic presentations of normal and abnormal ventricular function

- Detailed knowledge of the echocardiographic presentations of myocardial ischemia and infarction

- Knowledge of the echocardiographic manifestations of native valvular lesions and dysfunction

- Knowledge of the echocardiographic manifestations of cardiac masses, thrombi, cardiomyopathies, pericardial effusions, and lesions of the great vessels

- Detailed knowledge of the echocardiographic presentations of pulmonary, fat and air embolization

Interpersonal and communication skills:

- Ability to document echocardiographic results effectively in the patient's permanent medical record

- Discuss the basic findings of the TEE exam with the attending surgical specialist and critical care medicine physician involved in the patient's care

- Maintain a log of all TEE exams performed or performed and interpreted with a faculty member certified in advanced or basic PTE.

- Ability to recognize the situations where Call for help is beneficial to patient care. Help can be obtained from cardiologist, cardiac anesthesiologist or cardiac surgeon in such situations.

Professionalism:

- Demonstrate a commitment to professional responsibilities and adherence to ethical principles

- Interact with members of the surgery care team in a manner that reflects interpersonal integrity and behavior befitting an anesthesiologist

Practice-based learning and improvement:

Commitment to continuous improvement of basic echocardiography skills and correct deficiencies based upon the clinical experiences of the operating room and feedback from expert faculty.

Systems-based practice:

- Analyze the utilization, advantages and potential complications of intraoperative TEE monitoring and its role in determining further therapies.

- Actively participate in the quality improvement process of basic TEE monitoring.

Advanced Training

commitment. They are utilized in the cardiac operating room as hands on provider or they do night calls for the remaining 2 days of the week.

\section{Expectations for the First Month}

First week learning goal: One to one education on machine knobology and obtaining 2D basic TEE views will be provided during the first week to master the skills. Identification of normal cardiac structures and differentiation from artifacts and basics of different ultrasound modalities (2D, 3D, Color flow and spectral Doppler) will also be taught during this week.

Second week: All TEE examination should document estimation of left ventricular (LV) function (global, 
regional, systolic and diastolic), chamber size, LV mass estimation and right ventricle (RV) function to familiarize the trainee with ejection fraction estimation, volume estimation and different grades of diastolic dysfunction.

Third week: Examination of normal valves and evaluation for abnormal pathology of valves (regurgitation and stenosis) and evaluation of great vessels

Fourth week: Resident should be able to put all the information together and should be doing independent basic examination by themselves under faculty supervision.

\section{Typical TEE day at UPMC Presbyterian Hospital}

Transesophageal echocardiography residents arrive at 6.45 am and discuss preoperative echocardiography, planned operation and any contraindications to TEE like esophageal disease with the cardiac anesthesia faculty. Transesophageal echocardiography residents are responsible for entering the electronic order for TEE exam while the hands on provider for the patient starts the case and inserts lines. EKG connections to the TEE machine should be checked. Probe insertion takes place after intubation before the placement of central lines. Orogastric tube may be used to empty the stomach before probe insertion. Echo residents will help with surface ultrasound for arterial and venous cannulation. Transesophageal echocardiography examination with the faculty will follow the same sequence in each and every patient. Residents should be available for both pre- and post-bypass examinations. Pre- and postexamination can be taken as two different exams for the certification purposes (except in coronary artery bypass surgery) in valve and other pathologies. Transesophageal echocardiography resident also will participate in the TEE consultations for non-cardiac surgery and intensive care unit. Residents are responsible for documentation of TEE findings in the powernote (to be saved by the resident but will be verified and signed by the faculty).

\section{Second Month TEE Rotation}

By this time, they learnt their basics and should be motivated to complete their required numbers for basic certification. At the end of their rotation, the resident will meet with the director with the log of personally performed and observed TEE exams. The rotation director will provide the feedback, sign and forward the report and evaluation to the program director. Documentation of TEE log of cases should be done in the NBE required format. Resident also will take a multiple choice questions test with TEE images and videos to complete the rotation.
Table 6: Basic PTE lectures

Ultrasound and knobology
PTE certification
PTE safety guidelines
Conduct of a standard basic PTE examination
Evaluation of left ventricular function
Evaluation of right ventricular function
Basic evaluation of valves and valve pathology
Evaluation of hemodynamics by TEE and specific diagnosis
during hemodynamic instability

\section{Didactics}

All lectures on basic TEE are available online through intranet website of the department and residents are strongly advised to review all power-point presentations (Table 6) before completion of 2 months rotation. All TEE residents will get together once a week for a TEE review by a cardiac anesthesia faculty. Typically, five to $10 \mathrm{TEE}$ examination can be reviewed in a week and this helps to complete their reviewed TEE requirement for NBE certification.

\section{Advanced PTE Training for Cardiothoracic Anesthesia Fellows}

The application of an advanced perioperative TEE examination is to utilize the full diagnostic potential of perioperative TEE including direction of the perioperative surgical decision-making process. ${ }^{19}$ All basic principles will apply for advanced PTE training. In addition, there will be special requirements for advanced PTE training (Table 7). Residents who wish to proceed to CT Anesthesiology fellowship can get credit for their basic TEE numbers done during residency period as long as they are done in advanced cardiac surgery environment. ${ }^{22}$

Cardiothoracic anesthesiology fellows will need 2 months dedicated to echocardiography to obtain the necessary skills though it is variable in different institutions. Based on the surgical volume, one of those months can be spent in echocardiography laboratory to get exposure to various pathologies. Transthoracic studies reviewed by qualified echocardiographer will count towards the requirement for advanced PTE certification. ${ }^{22}$ Application of learnt skills to the patient management can be obtained while doing hands on anesthesiology rotation in the operating room.

Clinical training and didactics for CT anesthesiology fellowship should include advanced methods of TEE including epiaortic echocardiography (EUS), epicardial echocardiography in patients with contraindications to TEE, 3DTEE, tissue Doppler and advanced hemodynamic calculations. Advanced training in PTE should include 25 EUS interpretations; the individual trainee should 
Table 7: Advanced perioperative transesophageal echocardiography education goals

Patient care:

- All the technical skills defined under basic training

- Ability to acquire or direct the acquisition of all necessary echocardiographic data including epicardial and epiaortic imaging

- Ability to recognize subtle changes in segmental ventricular contraction indicative of myocardial ischemia or infarction

- Ability to quantify systolic and diastolic ventricular function and to estimate other relevant hemodynamic parameters

- Ability to quantify normal and abnormal native and prosthetic valvular function

- Ability to assess the appropriateness of cardiac surgical plans

- Ability to identify inadequacies in cardiac surgical interventions and the underlying reasons for the inadequacies

- Ability to aid in clinical decision making in the operating room

Medical knowledge:

- All the cognitive skills defined under basic training

- Detailed knowledge of the principles and methodologies of qualitative and quantitative echocardiography

- Detailed knowledge of native and prosthetic valvular function including valvular lesions and dysfunction

- Knowledge of congenital heart disease

- Detailed knowledge of all other diseases of the heart and great vessels that is relevant in the perioperative period

- Detailed knowledge of the techniques, advantages, disadvantages, and potential complications of commonly used cardiac surgical procedures for treatment of acquired and congenital heart disease

- Detailed knowledge of other diagnostic methods appropriate for correlation with perioperative echocardiography

Interpersonal and communication skills:

- Discuss the advanced comprehensive findings of the TEE exam with the attending cardiac anesthesiologist, cardiac surgeon and critical care medicine physician involved in the patient's care with progressive independence

- Demonstrate all skills described in the basic TEE goals

Practice-based learning and improvement:

- Learn from his/her clinical experiences in the OR for ongoing development and improvement of echo skills in a progressively independent manner

- Correct deficiencies in knowledge and expertise in TEE based upon feedback received from cardiac anesthesiology faculty

- Develop a plan for his/her continuing education in perioperative TEE to foster life-long learning and self-assessment

Systems-based practice:

- Analyze the utilization, advantage and potential disadvantages of intraoperative TEE and its role in determining further therapeutic measures, both medical and surgical

- Analyze the use of TEE to determine the best practices, use and cost effectiveness of this technology

- Actively participate in the quality improvement process in the division of cardiothoracic anesthesiology

personally direct five of them. ${ }^{10}$ They should also get adequate training in TEE guided cannulations during minimally invasive procedures, ventricular assist device (VAD) placement, transplantations, aortic surgery and extracorporeal membrane oxygenation support (ECMO). Adult congenital heart disease exposure is mandatory but pediatric echocardiography training is not a requirement of adult CT anesthesia fellowship.

\section{Examination and Certification}

National Board of Echocardiography administers several examinations related to the training in echocardiography and provides certification for physicians who completed clinical training. Those who completed only the examination without documentation of clinical training are given testamur status. NBE works in collaboration with ASE and SCA. Two examinations are administered in relation to perioperative echocardiography. Examination for special competence in basic perioperative TEE is administered every 2 years and examination for special competence in advanced perioperative TEE is administered every year. Any physician in training or practice can appear for the examination and receive testamur status. However, license to practice medicine, board certification and $\mathrm{CT}$ anesthesia fellowship training are required to obtain advanced TEE certification. Basic TEE certification does not require $\mathrm{CT}$ anesthesia fellowship training. Several anesthesiologists also complete the examination for special competence for clinical echocardiography, which also covers the transthoracic echocardiography, stress echocardiography and contrast echocardiography. Although this is meant for cardiology practice, transthoracic ultrasound is slowly gaining importance in the perioperative and critical care monitoring. PTE director should be aware of the procedures and requirements so that he can guide the younger generation in the right direction.

\section{RESEARCH IN PERIOPERATIVE ECHOCARDIOGRAPHY}

Promotion of clinical research is one of the major roles of perioperative echocardiography leadership. There is plenty of scope for research in perioperative echocardiography. 
First, the guidelines are established by evidence from non-randomized clinical trials and expert opinion. Validation of many of these existing guidelines should be done by proper randomized, prospective and multicenter clinical trials. Second, the high quality studies should be conducted on the relation between intraoperative TEE based decision-making of various pathologies and longterm patient outcomes. Third, the utility of newer imaging modalities like tissue Doppler and 3DTEE in various clinical situations should be evaluated. Transesophageal echocardiography education related research (e.g. the role of TEE simulation) should also be promoted to derive the best learning methods. Finally, creation of multicenter large databases will help to answer many questions as multicenter, randomized and prospective trials are not feasible and can be very expensive.

\section{CONTINUOUS QUALITY IMPROVEMENT}

Continuous quality improvement is an internal and departmental quality assessment project, which will evaluate the performance of an already established perioperative echocardiography service. ${ }^{23}$ Continuous quality improvement involves evaluation of equipment, review of performance and interpretation skills of echocardiographers, review of documentation of patient echocardiography reports and providing continuing education for the members to maintain quality. Echocardiography service director is primarily responsible for CQI process and documentation of CQI. First, all perioperative echocardiography team members should be aware of such a process and official policy manual should be distributed to all team members. Although ASE and SCA set recommendations for United States, all nations should work on country-specific guidelines for CQI.

\section{Equipment Evaluation}

Current requirements include evaluation of echocardiography machine, probes, digital storage and their maintenance. The machine should be capable of two dimensional, various Doppler capabilities (color and spectral Doppler) and M-mode echocardiography. Currently, 3D echocardiography is not a requirement and we will see this modality gain definite indications in the future. All TEE probes should be capable of multilane imaging. Echocardiography connections to the machine should be standard before conduct of any examination. Echocardiography services should be able to capture, store and review all the performed examinations in digital imaging and communications mode (DICOM). High speed and preferably wireless networking for speedy transfer of images to the permanent storage is also recommended.
Maintenance of equipment and probes including checking for current leakage should be performed periodically according to manufacturer's recommendations. Cleansing of machine, EKG cables and probes should be checked every few months. Proper maintenance of TEE equipment is cost saving considering the cost of purchasing new machine $(\$ 150,000-250,000)$ and probes $(\$ 20,000-50,000$ depending on the type of TEE probe). A fully organized echocardiography service should have wide array of probes meant to be used for TEE, TTE, surface ultrasound for cannulations, epiaortic and epicardial echocardiography. Perioperative echocardiography director works with the members of the PTE team, equipment in-charge of anesthesiology department and hospital equipment purchasing committee to make sure the equipment needs of the echocardiography service are fulfilled.

\section{Competence of Personnel}

While hiring the anesthesiologists with qualifications (testamur status and National Board of Echocardiography certification) is vital for the service, maintenance of their competence is also equally important. Critical factor is cardiac surgical patient volume per anesthesiologist, which will keep them in touch with the practice of echocardiography. A minimum of 25 PTE examinations should be performed in a month collectively by the echocardiography service. Although this is the minimum recommended number, ideally this requirement should depend on the number of anesthesiologists in a particular program. For individual echocardiographers, a minimum of 50 TEE examinations should be interpreted, of which 25 should be personally performed in a year. ${ }^{23}$ Clinical competence can also be evaluated by an independent review of the PTE examinations performed and reported by individual echocardiographers. It is recommended that five PTE examinations should be reviewed every year for any echocardiographer. Any discrepancy in interpretation should be reported back to the echocardiographer and evidence suggests that this feedback process helps in quality improvement. ${ }^{24,25}$ Currently, recertification examination in PTE has to be taken by the practicing echocardiographers every 10 years to maintain their certification.

\section{Continuing Education}

In addition to maintenance of practical competence, continuing education to update the recent advances in echocardiography is also a requirement. Continuing medical education (CME) to the members of echocardiography team can be arranged in multiple ways. ASE, SCA, European Society of Cardiology (ESC), European 
Association of Cardiothoracic Anesthesiologists (EACTA), Asian and Indian Society of cardiac anesthesia, Society of Transesophageal Echocardiography (STE) and other societies conduct echocardiography symposiums and conferences. Faculty members should attend one of those conferences to get their $\mathrm{CME}$ credits and updates. Interdepartmental Echocardiography Conferences within the hospital system can bring cardiologists; cardiac surgeons and cardiac anesthesiologists together for interactive problem based patient management discussions and also provide CME credits. Institutional Annual Echocardiography course is another way of providing CME credits and education to the members. Echocardiography director should take the lead in all above-mentioned roles. Echocardiography director also should encourage their faculty and trainees to publish interesting echocardiography reports and get involved in clinical research related to perioperative echocardiography. ASE/SCA CQI recommends that physicians in practice should maintain $15 \mathrm{CME}$ credits dedicated to echocardiography in 3 years. Recertification in PTE also requires $15 \mathrm{CME}$ credits in three years immediately preceding certification examination. ${ }^{23}$

\section{DOCUMENTATION}

Surgical team should mention the need for intraoperative TEE in the operating room (OR) schedule and an order should be placed in the electronic medical record (EMR) by the anesthesia team with the indication clearly stated. If TEE is done outside the OR, the physician requesting the exam should place an order in the medical record. Contraindications, risks and benefits should be discussed with the patient and documented. Consent obtained for the procedure and usually documented as a part of the anesthesia consent. After the examination is complete, a preliminary report should be generated immediately and complete report should be finalized by 24 hours. The report should correlate with the findings reported to the surgical team. If the trainee creates the TEE report, the qualified anesthesiologist should add clear addendum that he was present and supervised the examination. $\mathrm{He}$ also should document his agreement with the trainee's report. Currently, Society of Thoracic Surgeon's (STS) database also captures data from intraoperative TEE examination. Their specifications, such as changes in ventricular function, valvular function and aortic integrity should be clearly documented so that STS database can represent accurate outcomes.

The medicare and medicaid Electronic Healthcare record (EHR) Incentive Programs provide incentive payments to eligible professionals, eligible hospitals, and critical access hospitals (CAHs) as they adopt, implement, upgrade or demonstrate meaningful use of certified EHR technology. Adaptation of electronic documentation for TEE report is strongly recommended. Proper documentation improves communication within and between the hospital systems, patient care and quality. It also avoids the use of repetitive diagnostic tests for the same patient decreasing overall healthcare costs. Electronic documentation from multiple programs can be combined to generate large databases and the results of database research can be used to optimize patient outcomes. Finally, documentation is key for billing and reimbursement.

American society of echocardiography and ESC published guidelines for documentation of intraoperative echocardiography. ${ }^{26,27}$ All key components should be included in the documentation (Table 8). It is always helpful to document quantitative information, such as LV ejection fraction, tricuspid annular plane systolic excursion, vena contracta of mitral regurgitation jet and mean gradient across the stenosed mitral valve. Pressure environment of the operating room may not allow all quantitative measurements to be done in the prebypass period. It may not be necessary to repeat all measurements if it was done preoperatively by the cardiologists. Qualitative assessment of ventricular function and valvular function is acceptablein the hands of experienced echocardiographers. However, it is important to document quantitative information from the post-bypass period after optimization

Table 8: Key components of intraoperative TEE documentation

\author{
Demographic and patient identifying information \\ Patient: Name, Medical Record Number, Age, Sex, date of birth \\ Equipment: Machine ID, Probe ID, Imaging modalities (2D, 3D, Doppler, etc.) \\ Personnel: Anesthesiologist, Hands on provider, Surgeon, Echocardiographer, Trainee \\ Surgery and Indication: Procedure planned and procedure done, Any alteration in procedure based on TEE findings \\ Other: Location, date and time of procedure, quality of study (poor windows) \\ Prebypass standard TEE examination findings (Quantitative and qualitative) \\ Post intervention Relevant TEE findings (Quantitative and qualitative) \\ Comments to include any additional information (cannulations, shunts, etc.) \\ Complications if any \\ Attestation of faculty echocardiographer
}

Conclusive statement of the findings with possible description of pathophysiology. Therapeutic suggestions can be added 
of hemodynamics. It is also useful to document the actual hemodynamic values on the screen at the time of hemodynamic measurements. This will be the reference value against which the follow-up echocardiographic information from the cardiology laboratory will be compared. Documentation of gradient across the repaired or replaced valves, left ventricular ejection fraction after revascularization and documentation of dynamic LVOT gradients after septal myomectomy are few examples. A second cardiopulmonary bypass run is unavoidable in some patients. Documentation of the exact pump run in which the image was taken can also be useful.

As discussed in the TEE safety section, patients with significant esophageal disease or other relative contraindications should be documented along with the discussion of risk vs benefits of the TEE procedure. Any other precautions taken, such as thoracic surgical intervention (esophagoscopy, dilatation) before TEE probe insertion should also be mentioned. Complications are rare but can be severe and life threatening. Probe insertion difficulties and injuries should be explained in detail along with treatment done for the complications.

\section{BILLING AND REIMBURSEMENT}

Currently, billing for the echocardiography service does not mandate echocardiographer to be qualified by NBE as testamur or diplomate. Several experienced cardiac anesthesiologists use TEE without NBE certification after obtaining hospital privileges. It is up to the individual hospitals to allow anyone with adequate exposure and experience to perform, interpret and report TEE exams. Most of those anesthesiologists would have performed the required 300 TEE examinations in their experience. However, the outlook is changing and fresh graduates who intend to join the teams are mandated to obtain certification to perform TEE on cardiac surgical patients.

When the service is submitted for billing, billing (Current Procedure Terminology-CPT) and diagnostic codes (International Classification of Diseases-ICD) are the key factors in reimbursement. The billing codes are specific and described in Table 9. Most private party insurances follow the policy created by Center for Medicare Services (CMS). Transesophageal echocardiography probe insertion can be billed separately even if other specialties perform TEE for diagnosis. Transesophageal echocardiography as a part of anesthesia management for monitoring without appropriate medical necessity or a diagnostic criterion is not reimbursed currently. If there are great shifts in volume status or hemodynamic instability in a vulnerable patient population (patients with significant heart disease) or particular surgical population (liver transplantation), this can be billed using
93312 billing code. If anesthesiologist is called in to perform TEE on hemodynamically unstable patient during non-cardiac surgery, he should mention the diagnostic criteria and can bill it as a diagnostic TEE. Congenital heart disease, color flow and spectral Doppler all have separate codes for reimbursement.

Other pre-requisites for reimbursement include the order from surgical or any other requesting team for the need of TEE and proper reporting of TEE within 24 hours by the echocardiographer. If anesthesiologist who is performing TEE is also involved in the patient management, he should use a modifier to bill for the services. Though this is the process in United States, it is important for anesthesiologists learn the aspects of billing procedures in their individual countries and optimize the reimbursement for their services.

\section{FUTURE CHALLENGES}

It can be argued that basic TEE is a skillset that should be obtained by all anesthesiologists practicing at university medical center who treat sicker patients. Critically ill patients including trauma victims, vascular surgery and septic patients all will benefit from TEE directed hemodynamic therapy. However, training all anesthesiologists who are already in practice is a huge challenge. Instead, the focus should be on training the future generation and all training programs should introduce a structured TEE rotation in their residency programs.

Proper training in transthoracic ultrasound can have significant impact on perioperative patient management. ${ }^{28-30}$ Transthoracic echocardiography can be used to evaluate cardiac and valve function in high-risk patients presenting to preoperative holding area without previous cardiology evaluation. A quick TTE will answer several important questions and avoid unnecessary delays in getting the patients to the operating room. Transthoracic echocardiography can be used to evaluate hemodynamic instability intraoperatively in patients not intubated or in patients with contraindications for TEE. Transthoracic echocardiography is also very useful to quickly scan the heart and lungs in the postoperative unstable patients with hypotension or hypoxemia. Critical care physicians have developed protocols for the use of TTE in resuscitation and hemodynamic instability in ICU. ${ }^{31}$ As cardiac anesthesiologists are already trained in TEE, it is easier for them to learn basic TTE examination. There are efforts to introduce focused transthoracic ultrasound training for anesthesiologists but we will need proper training and practice guidelines before start using TTE in perioperative medicine. ${ }^{32,33}$

Newer echocardiographic imaging techniques, such as 3D echocardiography, tissue Doppler and strain imaging 
Table 9: Billing codes used for intraoperative transesophageal echocardiography

93312 Echocardiography, transesophageal, real time with image documentation (2D) (with or without M-mode recording); including probe placement, image acquisition, interpretation and report.

This service involves placement of the transesophageal probe, obtaining the appropriate images and views, and critical analysis of the data. Patients with increased risks of hemodynamic disturbances may require probe insertion and interpretation of the echocardiogram. This includes, but is not limited to, histories of congestive heart failure, severe ischemic heart disease, valvular disease, aortic aneurysm, major trauma and burns. It may also be indicated in certain procedures that involve great shifts in the patient's volume status. Such procedures may include vascular surgery, cardiac surgery, liver resection/ transplantation, extensive tumor resections and radical orthopedic surgery. The use of TEE may also be indicated when central venous access is contraindicated or difficult and it is not possible to adequately assess blood loss and replacement, impairment of venous return, and right and left heart function without the TEE.

93313 Echocardiography, transesophageal, real time with image documentation (2D) (with or without $\mathrm{M}$-mode recording); placement of transesophageal probe only.

Although the procedure is generally safe, the proper insertion of the probe requires skill and judgment. There are a few inherent risks to placement of the probe, including pharyngeal and/or laryngeal trauma, dental injuries, esophageal trauma, bleeding, arrhythmias, respiratory distress and hemodynamic effects. There have even been case reports of perioperative death attributed to TEE probe placement.

93314 Echocardiography, transesophageal, real time with image documentation (2D) (with or without M-mode recording); image acquisition, interpretation and report only.

This code is used when one physician inserts the probe and another physician interprets the images. Physicians who obtain and interpret cardiac images and provide a report but who did not place the TEE probe should use this code to report their service.

93315 Transesophageal echocardiography for congenital cardiac anomalies; including probe placement, image acquisition, interpretation and report.

This service involves placement of the transesophageal probe, obtaining the appropriate images and views, and critical analysis of the data in patients with congenital cardiac anomalies. This includes, but is not limited to, congenital valve problems, such as bicuspid aortic valve, septal defects, including patent foramen ovale, and more complicated congenital heart defects. This includes, but is not limited to, all the indications listed for code 93312, but in patients with congenital cardiac anomalies.

93316 and Similar to 93313 and 93314 respectively but for congenital diseases of the heart.

93317

93318 Echocardiography, transesophageal (TEE) for monitoring purposes, including probe placement, real time 2-dimensional image acquisition and interpretation leading to ongoing (continuous) assessment of (dynamically changing) cardiac pumping function and to therapeutic measures on an immediate time basis.

This code is used when the patient's condition, as described under 93312, requires repetitive evaluation of cardiac function in order to guide ongoing management.

93320 Doppler echocardiography, pulsed wave and/or continuous wave with spectral display

This code is used to evaluate blood velocity and flow patterns through various cardiac and vascular structures. Stenotic lesions generally lead to increased blood velocity proportional to the degree of stenosis, thereby providing a method to assess the severity of stenosis. Velocity measurements are also used to calculate the area of stenotic valves and regurgitant orifices. This code may be submitted along with code 93312 or 93315.

93321 Doppler color flow velocity mapping.

This code is used to evaluate the direction and character of blood flow through various cardiac and vascular structures. This code may be submitted along with code 93312 or 93315 .

$76998 \quad$ Ultrasound guidance, intraoperative

$76999 \quad$ Unlisted ultrasound procedure (e.g. diagnostic interventional)

Modifiers 59: If anesthesiologist performing TEE is also providing anesthesia care

29: If anesthesiologist does not own equipment but responsible for professional service and reporting

are being utilized in the operating room in specific circumstances at present and will have more applications in clinical patient management in future. Three-dimensional echocardiography is vital in making decisions on mitral valve lesions, valve repairs and prosthetic valve diseases. Practicing physicians at university hospitals not only have to learn these tools and implement them in patient care but also need to teach the future generation.

\section{SUMMARY}

Perioperative echocardiography has been proven to improve patient safety and healthcare quality in cardiac and noncardiac surgical patients. Perioperative echocardiography directed hemodynamic therapy involves teamwork of cardiac anesthesiologists, critical care physicians and cardiac surgeons with anesthesiologists leading the team. Perioperative echocardiography leadership is responsible for formulating guidelines for clinical practice, education and training, billing and reimbursement, equipment purchase and maintenance, continuing medical education, quality improvement and interdepartmental communication regarding echocardiography. Conduct of clinical research related to echocardiography and maintenance of echocardiography database are other 
components of perioperative echocardiography service and the multifaceted role of PTE leadership should be recognized at all tertiary healthcare institutions.

\section{REFERENCES}

1. Eltzschig HK, Rosenberger P, Löffler M, Fox JA, Aranki SF, Shernan SK. Impact of intraoperative transesophageal echocardiography on surgical decisions in 12,566 patients undergoing cardiac surgery. Ann Thorac Surg 2008;85(3): 845-852.

2. Fox J, Glas K, Swaminathan M, Shernan S. The impact of intraoperative echocardiography on clinical outcomes following adult cardiac surgery. Semin Cardiothorac Vasc Anesth 2005;9(1):25-40.

3. Shillcutt SK, Markin NW, Montzingo CR, Brakke TR. Use of rapid 'rescue' perioperative echocardiography to improve outcomes after hemodynamic instability in noncardiac surgical patients. J Cardiothorac Vasc Anesth 2012;26(3):362-370.

4. Suriani RJ, Neustein S, Shore-Lesserson L, Konstadt S. Intraoperative transesophageal echocardiography during noncardiac surgery. J Cardiothorac Vasc Anesth 1998;12(3):274-280.

5. Rapezzi C, Rocchi G, Fattori R, et al. Usefulness of transesophageal echocardiographic monitoring to improve the outcome of stent-graft treatment of thoracic aortic aneurysms. Am J Cardiol 2001;87(3):315-319.

6. Anguissola GB, Mangiarotti R, Pierini A, et al. Wall stress in the assessment of left ventricular function in surgery of abdominal aortic aneurysm. Validity and importance of transesophageal echocardiography (TEE) in intraoperative monitoring. Minerva Anestesiol 1994;60(5):237-244.

7. HüttemannE,SchelenzC,KaraF,ChatzinikolaouK, ReinhartK. The use and safety of transoesophageal echocardiography in the general ICU-a mini review. Acta Anaesthesiol Scand 2004;48(7):827-836.

8. Denault AY, Couture P, McKenty S, et al. Perioperative use of transesophageal echocardiography by anesthesiologists: impact in noncardiac surgery and in the intensive care unit. Can J Anaesth 2002;49(3):287-293.

9. Rosenberger P, Shernan SK, Löffler M, et al. The influence of epiaortic ultrasonography on intraoperative surgical management in 6051 cardiac surgical patients. Ann Thorac Surg 2008;85(2):548-53.

10. Glas KE, Swaminathan M, Reeves ST, et al. Council for Intraoperative Echocardiography of the American Society of Echocardiography; Society of Cardiova scular Anesthesiologists. Guidelines for the performance of a comprehensive intraoperative epiaortic ultrasonographic examination: recommendations of the American Society of Echocardiography and the Society of Cardiovascular Anesthesiologists; endorsed by the Society of Thoracic Surgeons. J Am Soc Echocardiogr 2007;20(11):1227-1235.

11. Marcelino PA, Marum SM, Fernandes AP, Germano N, Lopes MG. Routine transthoracic echocardiography in a general Intensive Care Unit: an 18 month survey in 704 patients. Eur J Intern Med. 2009;20(3):e37-42.

12. Marcelino P, Marum S, Fernandes AP, Lopes MG. [Transthoracic echocardiography for evaluation of hypotensive critically ill patient]. Acta Med Port. 2006;19(5):363-371.

13. Practice guidelines for perioperative transesophageal echocardiography. An updated report by the American Society of
Anesthesiologists and the Society of Cardiovascular Anesthesiologists Task Force on Transesophageal Echocardiography. Anesthesiology 2010;112(5):1-7.

14. Hahn RT, Abraham T, Adams MS, et al. Guidelines for performing a comprehensive transesophageal echocardiographic examination: recommendations from the American Society of Echocardiography and the Society of Cardiovascular Anesthesiologists. J Am Soc Echocardiogr 2013;26(9):921-964.

15. Lang RM, Badano LP, Mor-Avi V, et al. Recommendations for cardiac chamber quantification by echocardiography in adults: an update from the American Society of Echocardiography and the European Association of Cardiovascular Imaging. Eur Heart J Cardiovasc Imaging. 2015;16(9):233-270.

16. Zoghbi WA, Chambers JB, DumesnilJG, etal. American Society of Echocardiography's Guidelines and Standards Committee; Task Force on Prosthetic Valves; Recommendations for evaluation of prosthetic valves with echocardiography and doppler ultrasound. J Am Soc Echocardiogr 2009;22(9): 975-1014.

17. Lang RM, Badano LP, Tsang W, et al. American Society of Echocardiography; European Association of Echocardiography. EAE/ASE recommendations for image acquisition and display using three-dimensional echocardiography. Eur Heart J Cardiovasc Imaging 2012;13(1):1-46.

18. Hilberath JN, Oakes DA, Shernan SK, Bulwer BE, D'Ambra MN, Eltzschig HK. Safety of transesophageal echocardiography. J Am Soc Echocardiogr 2010;23(11):1115-1127.

19. Reeves ST, Finley AC, Skubas NJ, et al. Council on Perioperative Echocardiography of the American Society of Echocardiography; Society of Cardiovascular Anesthesiologists. Basic perioperative transesophageal echocardiography examination: a consensus statement of the American Society of Echocardiography and the Society of Cardiovascular Anesthesiologists. J Am Soc Echocardiogr. 2013;26(5):443-456.

20. Ferrero NA, Bortsov AV, Arora $\mathrm{H}$, et al. Simulator training enhances resident performance in transesophageal echocardiography. Anesthesiology. 2014;120(1):149-159.

21. Matyal R, Mitchell JD, Hess PE, et al. Simulator-based transesophageal echocardiographic training with motion analysis: a curriculum-based approach. Anesthesiology 2014; 121(2):389-399.

22. Cahalan MK, Stewart W, Pearlman A, et al. Society of Cardiovascular Anesthesiologists; American Society of Echocardiography Task Force. American Society of Echocardiography and Society of Cardiovascular Anesthesiologists task force guidelines for training in perioperative echocardiography. J Am Soc Echocardiogr 2002;15(6):647-652.

23. Mathew JP, Glas K, Troianos CA, et al. Council for Intraoperative Echocardiography of the American Society of Echocardiography. ASE/SCA recommendations and guidelines for continuousquality improvement in perioperative echocardiography. Anesth Analg.2006;103(6):1416-1425.

24. Mathew JP, Fontes ML, Garwood S, et al. Transesophageal echocardiography interpretation: a comparative analysis between cardiac anesthesiologists and primary echocardiographers. Anesth Analg 2002;94(2):302-309.

25. Miller JP, Lambert AS, Shapiro WA, Russell IA, Schiller NB, Cahalan MK. The adequacy of basic intraoperative transesophageal echocardiography performed by experienced anesthesiologists. Anesth Analg 2001;92(5):1103-1110.

26. Feneck R, Kneeshaw J, Fox K, et al. European Association of Cardiothoracic Anaesthesiologists (EACTA) and the European 
Association of Echocardiography (EAE). Recommendations for reporting perioperative transoesophageal echo studies. Eur J Echocardiogr. 2010;11(5):387-393.

27. Available at: https://www.scahq.org/files/Report_of_Task_ Force.pdf (Accessed on 05 May 2015)

28. Canty DJ, Royse CF, Kilpatrick D, Bowman L, Royse AG. The impact of focused transthoracic echocardiography in the pre-operative clinic. Anaesthesia 2012;67(6):618-625.

29. Cowie B. Three years' experience of focused cardiovascular ultrasound in the peri-operative period. Anaesthesia 2011; 66(4):268-273.

30. Cowie BS. Focused transthoracic echocardiography in the perioperativeperiod. Anaesth IntensiveCare 2010;38(5):823-836.
31. Via G, Hussain A, Wells $M$, et al. International Liaison Committee on Focused Cardiac UltraSound (ILC-FoCUS); International Conference on Focused Cardiac UltraSound (IC-FoCUS). International evidence-based recommendations for focused cardiac ultrasound. J Am Soc Echocardiogr 2014;27(7):683.e1-683.e33.

32. Brakke TR, Desjardins G, Shillcutt SK, Vezina DP, Montzingo CR. Transthoracic echocardiography: training options for practicing physicians. J Cardiothorac Vasc Anesth 2011;25(5):e42-43.

33. Manecke GR Jr, Vezina DP. Perioperative transthoracic echocardiography: 'universal acid'? J Cardiothorac Vasc Anesth 2009;23(4):447-449. 